\title{
Nuevas adiciones al catálogo de Bernardo Lorente Germán
}

\author{
Jesús Porres Benavides \\ Universidad Rey Juan Carlos \\ jesus.porres@urjc.es
}

En los últimos años estamos asistiendo a un interés creciente por la pintura sevillana que nos está permitiendo profundizar en el conocimiento de unos pintores poco valorados en tiempos recientes como son los denominados murillescos del siglo XVIII. Efectivamente, una serie de pintores desde finales del siglo XVII y durante todo el XVIII copiaron y supieron adaptar los prototipos de Murillo. Entre estos artistas destacan Esteban Márquez, Juan Simón Gutiérrez y más adelante Alonso Miguel de Tovar, Domingo Martínez, Juan Ruiz Soriano o el propio Bernardo Lorente Germán (Navarrete, 2017: 19), considerados murillescos de segunda generación ${ }^{1}$ pero no con menor fortuna.

Lorente Germán es una de las figuras que mayor interés ha despertado en los últimos años ${ }^{2}$ gracias en parte a la aparición de obras suyas en el mercado o su puesta en valor tras restauraciones. Han reaparecido una serie de obras muy interesantes, algunas de ellas firmadas y asimismo se le ha restituido la paternidad de obras que se consideraban de otro autor, como la Anunciación de la capilla de la Fábrica de Tabacos (Porres, 2016: 190) anteriormente atribuida a Diego Bejarano (Rodríguez-Pantoja 1992: 196). Tal y como señala Quiles, se han publicado de Lorente numerosas obras, «algunas claramente suyas, otras erróneamente atribuidas» (2010: 211). Desgraciadamente, otras pinturas se han perdido como la Dolorosa del seminario de Baeza fechada en 1727 que fue descubierta por Pueyo en 1918 y se supone se destruyó en los sucesos de 1936. José Julia Sanfeliú estudió también en 1918 un santo Tomas de Aquino en el mismo edificio que parece que estaba firmado (1918: 28) ${ }^{3}$.

Algunas veces se han atribuido erróneamente obras de Lorente a otros autores. Por ejemplo, un cuadro que tiene mucho interés al encontrarse en una relevante colección británica (Pollok House), es un san Juanito con el cordero ${ }^{4}$ que ha sido catalogado en unos casos como obra de Alonso Miguel de Tovar (1678-1758) ${ }^{5}$ y en otros como «después de Bartolomé Esteban Murillo» o imitador. Ignacio Cano ya lo catalogó como posible obra de Lorente y se sabe que perteneció a la colección de Louis Philippe hasta 1853. Hacía pareja con un Niño Jesús en paradero desconocido ${ }^{6}$. La pintura toma el modelo del San Juanito con el cordero de Murillo existente en la National Gallery de Londres ${ }^{7}$, aunque con ligeras variantes como el celaje, el paisaje y por supuesto la imagen del santo niño, prototípica de Lorente y que no alcanza la maestría de Murillo ${ }^{8}$.

Otra obra que ha salido al mercado recientemente es una Divina Pastora atribuida al maestro, pero habría que ponerla en cuarentena a pesar de estar firmada ${ }^{9}$ y quizás atribuirla a un desconocido pintor sevillano del siglo XVIII del que se conocen otras obras del mismo tema en colecciones particulares $^{10}$.

Igualmente ha salido recientemente a la venta una Virgen con Niño, flanqueada por una corte celestial ${ }^{11}$ en este caso sí atribuible a Lorente [1]. En realidad, se trata de una interpretación iconográfica de la Virgen de todos los Santos de la iglesia de Ómnium Sanctorum de Sevilla rodeada de santos masculinos. En el grupo de la izquierda esta san Cristóbal, santo Domingo de Guzmán, san Bernardo, san Lorenzo, san Antonio y san Pablo y en el de la derecha san Pedro, san José, san Juan Bautista y otros.

Una pequeña pero interesante obra se ha exhibido en el mercado madrileño bajo el título El cónclave de los dominicos $^{12}$ [2] y ha sido catalogada como del siglo XVII. En realidad, es una obra posterior, de hacia 1730 y con una clarísima atribución a Lorente. Tampoco el tema iconográfico está descrito correctamente, pues parece más claro un momento de la vida de san Bernardo, monje cisterciense francés y abad de la abadía de Claraval. Este pudiera ser 


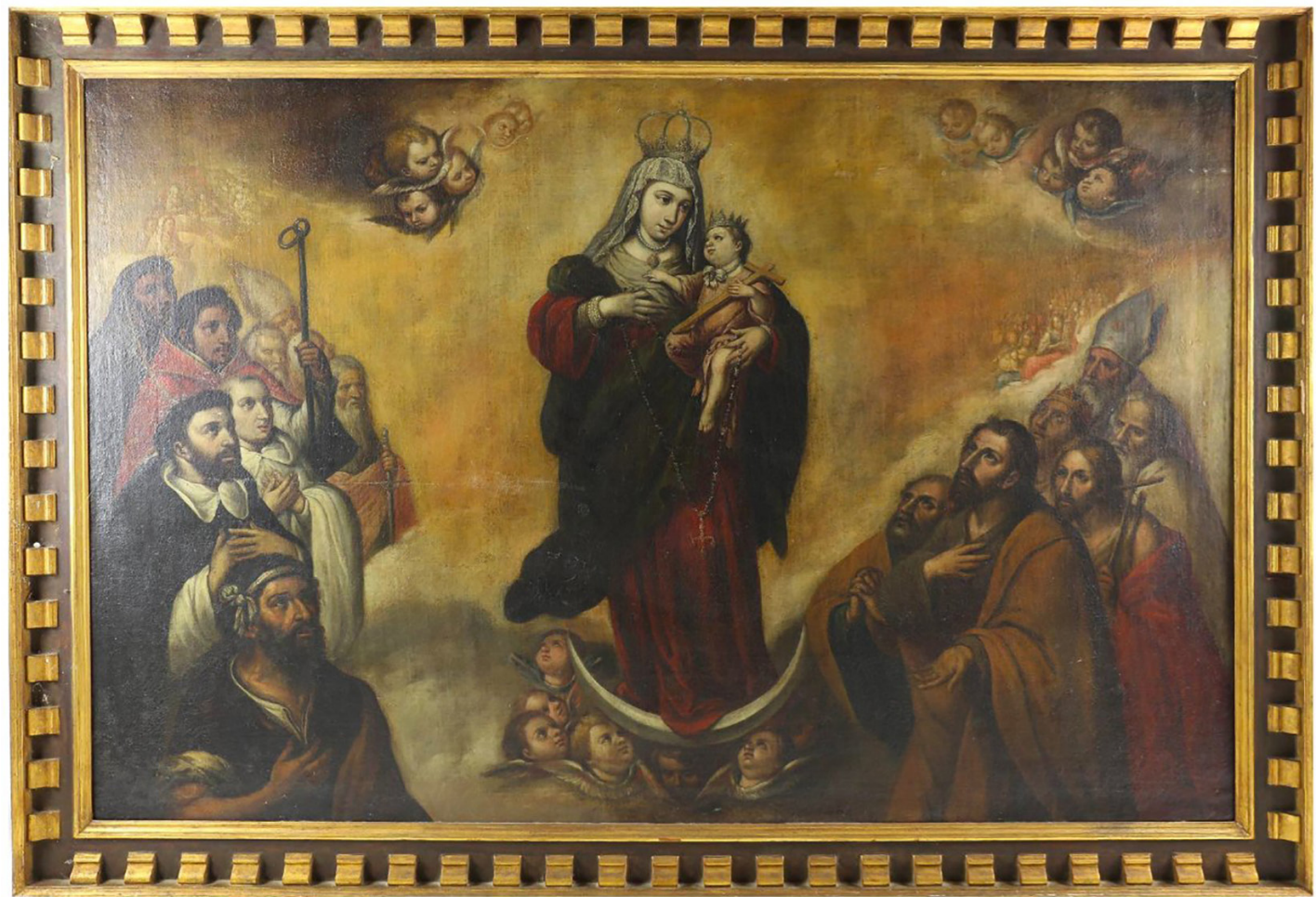

1. Virgen de todos los Santos, círculo de Bernardo Lorente Germán, $2 .^{\circ}$ tercio del siglo XVIII. Bonanova subastas

quizás el Concilio de Estampes, convocado por rey francés Luis VI en el año 1130, en el cual los padres se comprometieron a aceptar el arbitrio de san Bernardo en cuanto a la concurrencia al pontificado entre Anacleto e Inocencio II. Es una composición apaisada en torno a la cual se distribuyen los clérigos conversando en animado debate. San Bernardo está presidiendo el cónclave o reunión justo en el momento en el que va a intervenir, sentado delante de una mesa cubierta por un tapete encima de la cual se encuentran libros y tinteros. Junto a él, en un estrado, está sentado posiblemente el rey Luis VI, con los atributos regios que le corresponden, la capa de armiño con las flores de lis, el cetro y la corona propias de la monarquía francesa.

Esta pintura se puede relacionar con el San Bernardo de Claraval/13 dado a conocer hace unos años (Porres, 2016: 187) donde aparece la figura del santo en tres cuartos con atributos pasionistas como son la cruz, una caña con hisopo, una fusta, una corona entre sus manos, que es lo que se suele denominar como Arma Christi. El rostro y en parti- cular el tratamiento que hace de los ojos recuerdan a otras obras del autor.

Otra obra de reciente aparición que también podría asignarse a Lorente es un pequeño lienzo atribuido en el catálogo de la subasta al círculo de Bartolomé Esteban Murillo y denominado Virgen con el Niño, Santa Isabel y San Juanito ${ }^{14}$ [3]. Como recoge bien la información en el catálogo, sigue el modelo del cuadro del mismo título que se conserva en el Museo del Louvre Sagrada Familia también Ilamado La Virgen de Sevilla, aunque con pequeñas variantes. El cuadro de Murillo es más luminoso en general y en esta obra Lorente ha añadido al fondo y como en penumbra las figuras de santa Ana y Zacarías. También es algo diferente la actitud de santa Isabel con san Juan, que en el original de Murillo está más girada hacia el grupo del niño con su madre. Lorente tiene otras obras con esta iconografía como La Sagrada Parentela que firma hacia 1748, que ya dio a conocer Angulo en la colección Arenzana de Madrid en $1975^{15}$. 


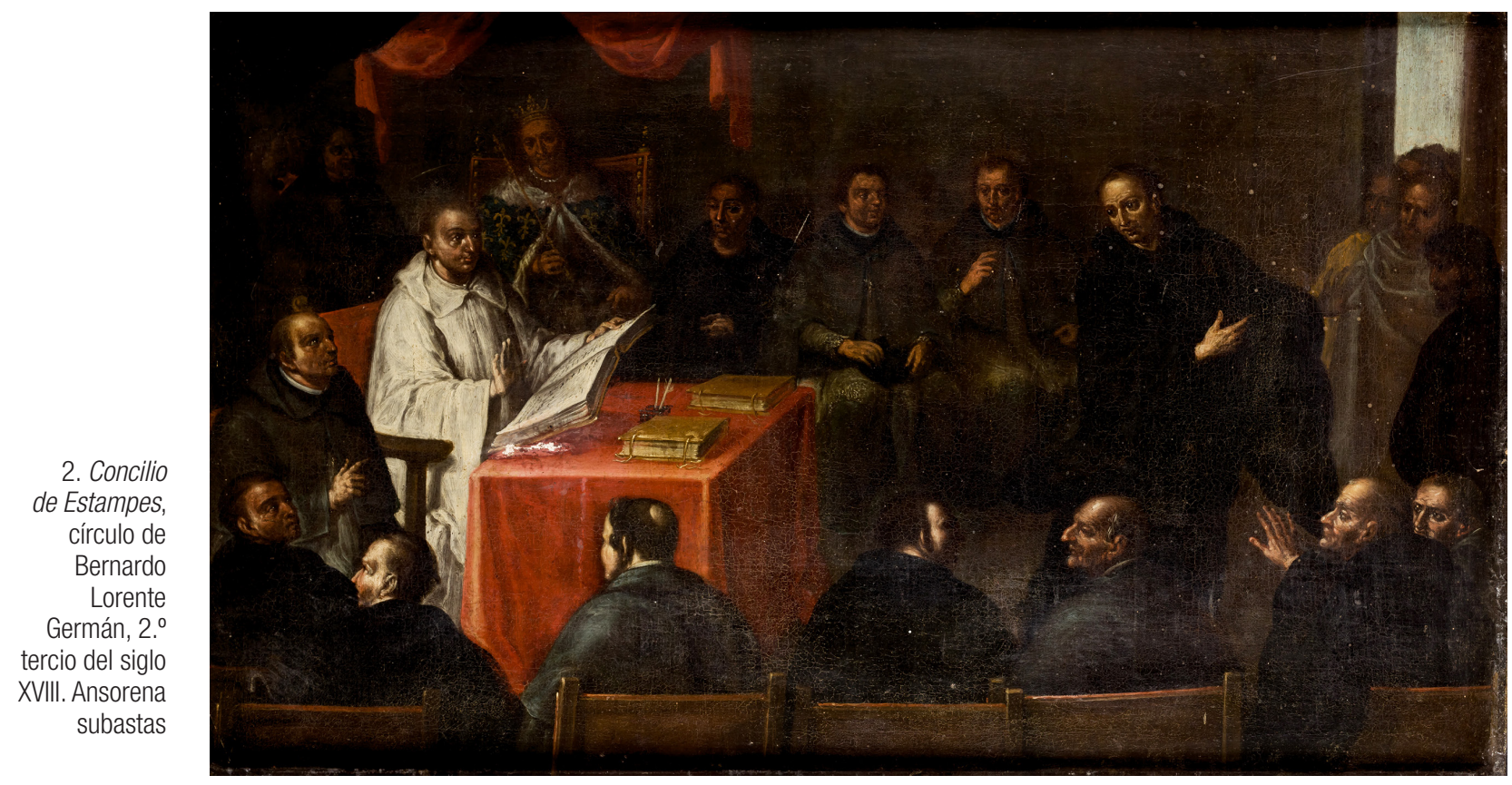

3. Sagrada Familia, círculo de Bernardo Lorente Germán, $2{ }^{\circ}$ tercio del siglo $\mathrm{XVIII.} \mathrm{Ansorena} \mathrm{subastas}$

En las redes sociales se ha publicado igualmente una Asunción de Santa Gertrudis, monja benedictina cisterciense, con gran similitud iconográfica a la subida al cielo de la Virgen María. Esta obra se encuentra firmada «Bernardus Lorente Germanus Facebat anno 1734» [4]. Gertrudis está representada como benedictina, vestida con el hábito negro, con un corazón en el pecho en el que está el Niño Jesús. La santa es transportada por siete ángeles que la llevan con una teatralidad muy barroca, mientras ella con los brazos abiertos parece estar ya contemplando a Dios que la va a acoger. Es una obra donde las tonalidades negras del hábito de la santa contrastan grandemente con las coloristas túnicas y mantos de los ángeles con colores bermellones, azules, ocres o las mismas alas.

Lorente Germán tiene otra obra de composición parecida, en la que representa a la Virgen en el cielo que se

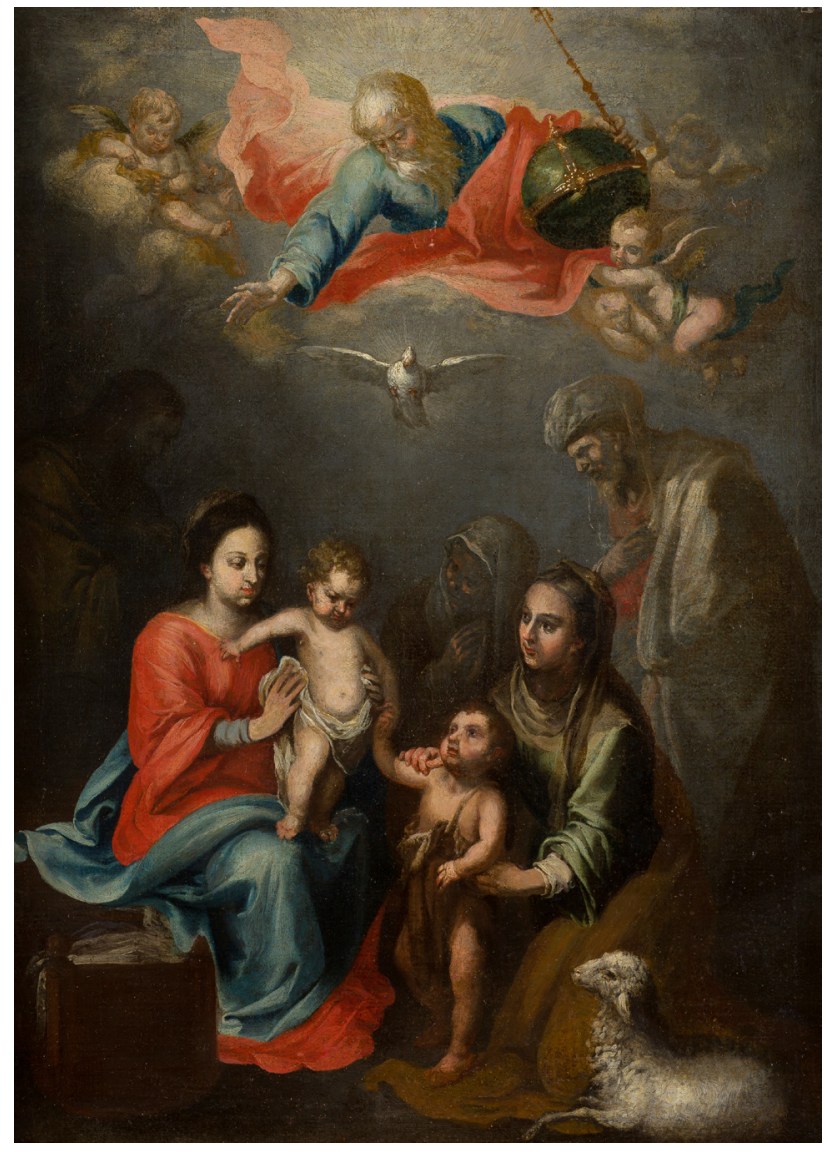




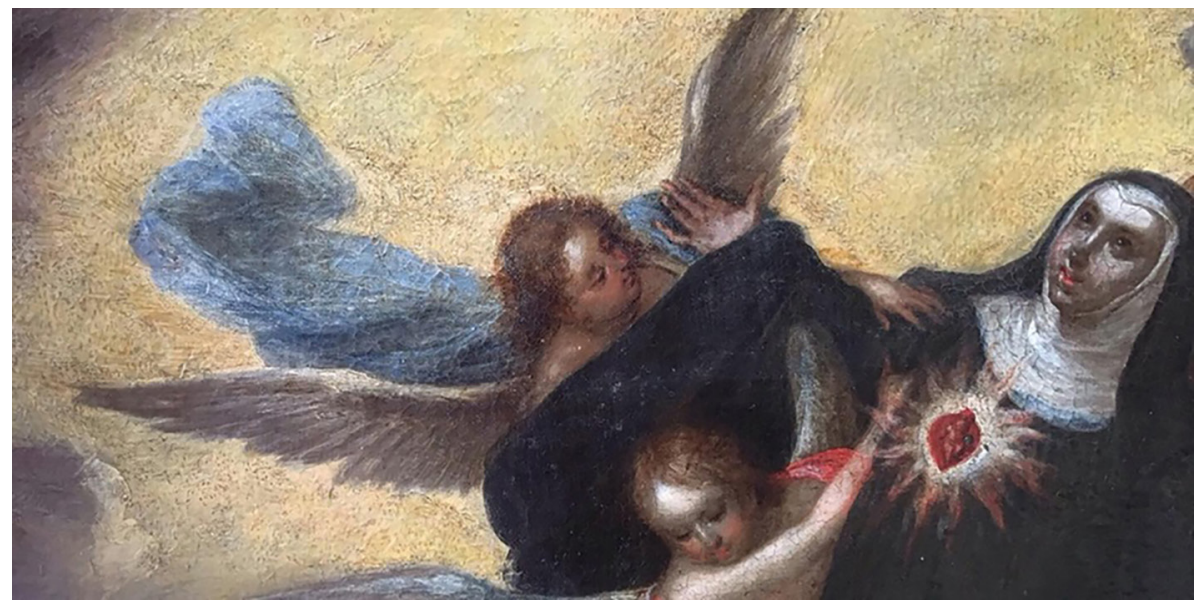

4. Asunción de Santa Gertrudis (detalle), Bernardo Lorente Germán, firmado y fechado en 1734

aparece a san Felipe Neri y que también estaba firmada y fechada (Quiles y Cano, 2006: 244) y se vendió en la sala Parés en 1959; actualmente está en paradero desconocido ${ }^{16}$. Por último, debemos mencionar otra obra de Loren- te German que ha sido localizada en la iglesia de Santiago de Llerena (Badajoz) y representa una aparición de la Virgen con el niño a san Juan Nepomuceno. Está fechado y firmado en 1749 .

\section{Notas}

1 Álvaro Cabezas los denomina con indudable acierto «murillistas», pues «así como los murillescos eran esos artistas que conocieron a Murillo y expandieron su estilo a través de los años», los murillistas «englobaban un espectro mayor y más avanzado en el tiempo» (Cabezas, 2019).

2 Obra fundamental fue Bernardo Lorente Germán y la pintura sevillana de su tiempo (1680-1759). Después se ha enriquecido con la aparición de las siguientes obras La influencia de Murillo en Bernardo Lorente Germán: Nuevas obras y Obras inéditas de Bernardo Lorente Germán. Fundamentales han sido también La escuela de Murillo. Aportaciones al conocimiento de sus discípulos y seguidores y Murillo y su estela en Sevilla.

3 Agradezco a José Joaquín Quesada la información.

4 Mide 80,6 x 60,3 cm, óleo sobre lienzo. Diego Angulo en el catálogo de Murillo (1981: 270), lo ve como una copia de época moderna y comenta «La diferencia de tamaño no permite la identificación».

5 Así aparece en las fichas de la Witt Library (Quiles y Cano, 2006: 256).

6 Este se subastó en Keir y estaba con el núm. 170 de catálogo.

7 Encargada por Justino de Neve, después legada a la iglesia de Santa María la Blanca en Sevilla y posteriormente incautada por las tropas francesas y luego pasó al museo en 1840. Mide $165 \times 106 \mathrm{~cm}$.

8 En otro museo de Glasgow, la Hunterian Art Gallery, tienen otra versión de Jesús como Buen Pastor que popularizara Murillo y adquirido por el Dr. William Hunter en una fecha tan temprana como 1783.

9 Subastas Isbiya, 10 de marzo de 2020, medidas: 93 × 61 cm. Agradecemos la información prestada.

10 Porres, 2017: 45

$11115 \times 167 \mathrm{~cm}$. Bonanova subastas.

12 Subastas Ansorena. 1. a sesión 5 de noviembre de 2019, con el lote num. 135. Tiene unas medidas de 51 x 85,5 cm. Agradecemos a Belén Puente la información prestada.

13 La pintura se vendió en Ansorena en mayo de 2012

14 La obra salió con el número 125 con unas medidas de 56 × 41 cm.

15 Con unas medidas de $109 \times 141 \mathrm{~cm}$ (Quiles y Cano, 2006: 238).

16 Medida $93 \times 65 \mathrm{~cm}$ 


\section{Bibliografía}

ANGULO, Diego (1981), Catálogo de Murillo; Murillo: Su vida, su arte, su obra, Catálogo crítico, vol. 2, Madrid, n. ${ }^{\circ} 335$,

CABEZAS GARCíA, Álvaro (2019), «En la senda de Murillo», en ROMÁN VILLALÓN, Álvaro (coord.), En la senda de Murillo: Tovar y la Divina Pastora, Catálogo de la exposición, Diputación Provincial de Huelva, Huelva.

JULIA SANFELIÚ, José (1918), «La Dolorosa de German Llorente» en Don Lope de Sosa. Crónica mensual de la provincia de Jaén, n. 61 , pp. 26-29.

NAVARRETE PRIETO, Benito (2017), "Murillo y su estela en Sevilla: imagen dialéctica y memoria anacrónica», en Murillo y su estela en Sevilla, Catálogo de la exposición, Sevilla.

PORRES BENAVIDES, Jesús (2019), «La influencia de Murillo en Bernardo Lorente Germán: Nuevas obras», Revista Quiroga, pp. 60-71.

- (2016), «Obras inéditas de Bernardo Lorente Germán», Archivo Español de Arte, vol. 89, n. 354, pp. 183-193.

- (2017), «La iconografía de la Virgen como Divina Pastora en la pintura sevillana del siglo XVIIl», Ensayos: Historia y Teoría del Arte, vol. 21, n. ${ }^{\circ} 33, \mathrm{pp} .37-59$.

QUILES GARCÍA, Fernando (2010), «Trampantojos sevillanos del siglo XVIIl», Atrio, n. ${ }^{\circ}$ 15-16, pp. 209-212.

QUILES GARCÍA, Fernando y CANO RIVERO, Ignacio (2006), Bernardo Lorente Germán y la pintura sevillana de su tiempo (1680-1759), Fernando Villaverde Ediciones, Madrid.

RODRÍGUEZ-PANTOJA MÁRQUEZ, Miguel (1992), Patrimonio artístico y monumental de las universidades andaluzas, Universidad de Sevilla, Sevilla.

VALDIVIESO, Enrique (2018), La escuela de Murillo. Aportaciones al conocimiento de sus discípulos y seguidores, Universidad de Sevilla, Sevilla. 